\title{
Trajectory-Based Hierarchical Adaptive Forwarding in Vehicular Ad Hoc Networks
}

\author{
Hao Wang, ${ }^{1}$ Liangyin Chen, ${ }^{1}$ Shijia Liu, ${ }^{1}$ Songtao Fu, ${ }^{1}$ Qian Luo, ${ }^{2}$ \\ Feng Yin, ${ }^{3}$ Limin Sun, ${ }^{4}$ and Zhanghua $\mathrm{Li}^{5}$ \\ ${ }^{1}$ School of Computer Science, Sichuan University, Chengdu 610225, China \\ ${ }^{2}$ The Second Research Institute of General Administration of Civil Aviation of China, Information Technology Branch, \\ Chengdu 610042, China \\ ${ }^{3}$ School of Computer Science and Technology, Southwest University for Nationalities, Chengdu 610041, China \\ ${ }^{4}$ Institute of Information Engineering, Chinese Academy of Sciences, Beijing 100864, China \\ ${ }^{5}$ Department of Engineering Physics, Institute of Public Safety Research, Tsinghua University, Beijing 100084, China
}

Correspondence should be addressed to Zhanghua Li; zh-li15@mails.tsinghua.edu.cn

Received 25 August 2016; Revised 7 November 2016; Accepted 19 December 2016; Published 11 January 2017

Academic Editor: Stephane Evoy

Copyright ( 2017 Hao Wang et al. This is an open access article distributed under the Creative Commons Attribution License, which permits unrestricted use, distribution, and reproduction in any medium, provided the original work is properly cited.

\begin{abstract}
This paper proposes a Trajectory-Based Hierarchical Adaptive Forwarding (THAF) scheme, tailored and optimized for the efficient multihop vehicle-to-vehicle (v2v) data delivery in vehicular ad hoc networks. We utilize the trajectories of vehicles provided by GPS-based navigation systems to predict forward delay and access area in a privacy-preserving manner. Different from existing trajectory-based forwarding schemes, we establish a hierarchical VANET topology to optimize forwarding path and adopt adaptive diffusion strategy to forward data in light-traffic situations. Through theoretical analysis and extensive simulation, it is shown that our design performs better than the existing schemes.
\end{abstract}

\section{Introduction}

Vehicular ad hoc networks (VANETs) are in a promising outlook in road safety, trip planning, and mobile Internet service.

Nowadays, cellular network (e.g., 4G-LTE) plays an important role in data delivery. However, to support various vehicular services with cellular network will occupy plenty of resources and will cost a lot to build and maintain quite a large number of base stations. So, there is a need to investigate a specialized wireless communication network for road network services.

Thanks to Dedicated Short Range Communications (DSRC) standardized by IEEE standards association, such as IEEE 802.11p [1] and GPS-based navigation services, data forwarding in vehicular networks has a promising future. In VANET, data forwarding mainly includes the following three ways: vehicle-to-infrastructure (V2I) communication; infrastructure-to-vehicle (I2V) communication; and vehicle-to-vehicle (V2V) communication. And there are many researches on these communication problems. Among them, the trajectory-based data forwarding scheme enjoys lower forwarding delay and higher success ratio [25]. TBD [4], which establishes the foundation of trajectorybased V2I communication, provides some useful models for delay estimation of V2I communication schemes. For I2V communication, TSF [2] and our former work TOAF [5] use trajectory-based stochastic models to decrease forwarding delay and enhance success ratio. However, what all these works missed are the schematization of forwarding path from a global perspective and adjustable forwarding strategy according to different traffic conditions (i.e., the density of traffic flow and data flow). For instance, when in light-traffic conditions, users may want to forward data to remote areas as quickly as possible. In this case, our diffusion strategy can be chosen to enhance the speed of data forwarding. Also, because all the existing trajectory-based models are based on stochastic models whose forwarding paths are solely determined by calculated forwarding delay, there is a possibility that all forwarding paths are congested in a single path 


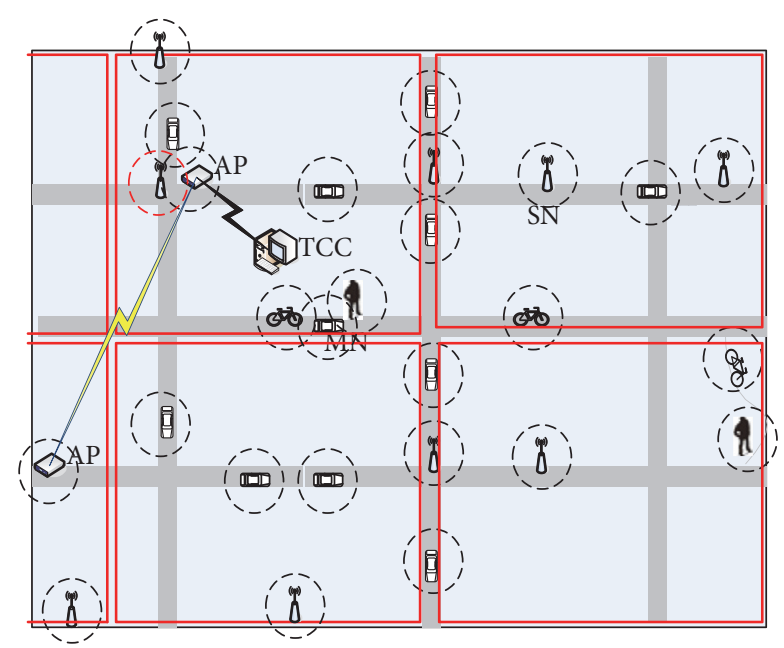

FIgURE 1: The model hypothesis sketch.

and it will cause severe delivery jitter due to the limitation of the bandwidth and signal channel. Highly motivated by these concerns, we have established a hierarchical VANET topology to optimize forwarding path.

The rest of this paper is organized as follows: Section 2 describes models used in our scheme including basic assumptions, receiving area prediction, network topology, and path arrangement model and diffusion strategy model. Section 3 explains the THAF protocol. Section 4 evaluates our design. And we conclude this paper in Section 5.

\section{Materials and Methods}

In this section, we will describe our models for THAF.

2.1. Assumptions. This work is based on the following set of assumptions on the road network and vehicle settings.

(1) Traffic Control Center (TCC) is a trustable entity that maintains the trajectories of all the vehicles without exposing to other uncorrelated vehicles for privacy concerns in the network. Also, the whole network topology will be integrated in TCC. As shown in Figure 1, TCC and APs are interconnected with each other directly through wired cables. TCC will initialize a preliminary forward path according to the delay estimation and network topology, which will be described later in Sections 2.2 and 2.3.

(2) Access Point (AP) which contains DSRC device, storage, and processor provides vehicles with wired network connectivity. For the cost effectiveness, they are sparsely deployed into road networks with their geographical location information available to vehicles. They interconnect with each other through wired cables.

(3) Stationary Node (SN) is the data keeper with DSRC device, storage, and processor without wired network connectivity to APs. SNs can also be described as relay nodes to support data forwarding from source node to destination node. As shown in Figure 1, each subregion (the red square) must have at least one $\mathrm{SN}$ responsible for data forwarding and storage in the region. And every four subregions get a father node (the SN with red circle beside AP) which can gather and disseminate data in these regions. We will articulate it later in Section 2.3.

(4) Mobile Node (MN) is installed in vehicles. It can carry and forward data to SNs, other MNs, and APs with DSRC device. Additionally, MNs can calculate their future trajectories information and report them to TCC via APs with SADV [6] or TSF [2] by inputting the destination when they start to move. MNs receive traffic statistics by the commercial navigation service [7]. Meanwhile, every MN has the ability to calculate the data forwarding delay from its current position to a certain area with the traffic statistics and shares the delay with other nodes for the purpose of selecting the next node to carry and forward data.

2.2. Model for Receiving Area Prediction. In order to forward data to the target vehicle, we should firstly calculate the Expected Delivery Delay (EDD) and Time-to-Live (TTL). EDD is the data forwarding delay estimation from the current source node to the potential receiving area and TTL is the delay estimation of the target vehicle from its current place to that potential receiving area under its trajectory.

To calculate EDD, it is necessary to build a preliminary path for data transmission from the source node $S$ to the destination node $D$ (the way to build it will be discussed in Section 2.3). Suppose there are $K$ intersections along the path between $S$ and $D$, and intersection $m(m \in[1, K])$ has $N_{m}$ neighboring intersections including intersection $m-1$ and $m+1$ along the preliminary transmission path. $j$ denotes the $j$ th neighboring intersection of $m\left(j \in\left[1, N_{m}\right]\right)$. As an example, Figure 2 shows one possible transmission section from $S$ to $D$.

Note that there are two approaches to transmit data. The first approach is to carry the data by the vehicle along its trajectory. The second approach is to deliver the data to another vehicle. To simplify the transmission problem, it is better to decompose the entire transmission process into two parts. The first part is to carry the message to intersection $m$ by the original vehicle. And the second part is to deliver the message to one of the neighboring intersections of $m$.

$P_{S, m}^{c}$ denotes the probability for a vehicle carrying the message from $S$ to $m$. $C_{S, m}$ denotes the expected carrying delay for the original vehicle to carry from $S$ to $m$. $D_{m, D}$ denotes the forwarding delay from $m$ to $D$ through any of the neighboring intersections of $m$. Finally, the Expected Delivery Delay can be computed as

$$
\mathrm{EDD}=\sum_{m=1}^{K}\left(P_{S, m}^{c} \times\left(C_{S, m}+E\left(D_{m, D}\right)\right)\right) .
$$

Note that $P_{S, m}^{c}$ can be calculated as multiples of $P_{h, h+1}^{c}$, which is the probability of the original vehicle carrying the 


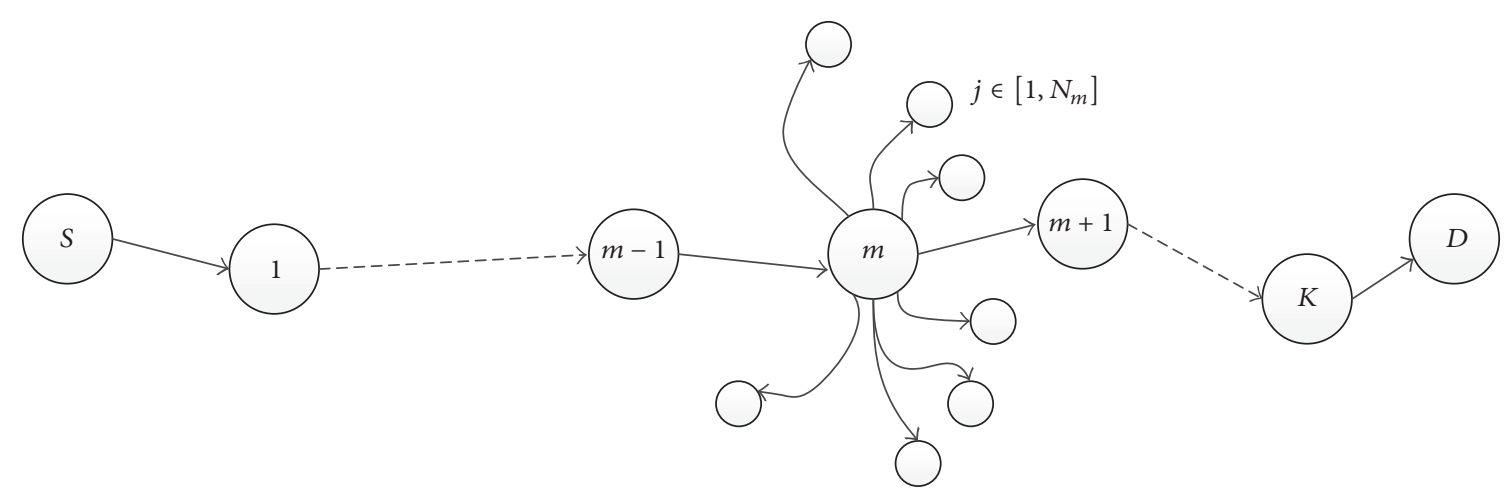

FIgURE 2: A transmission section model for time estimation.

message from intersection $h(h \leq m-1)$ to intersection $h+1$ along the preliminary path. So $P_{S, m}^{c}=\prod_{h=0}^{m-1} P_{h, h+1}^{c}$. And the expected value of the forwarding delay from $m$ to $D$ can be computed as $E\left(D_{m, D}^{j}\right)=\sum_{j=1}^{N_{m}} P_{m}^{j} D_{m, D}^{j}$, where $P_{m}^{j}$ denotes the probability for the carrying vehicle to choose intersection $j$ to forward data and $D_{m, D}^{j}$ denotes the expected forwarding delay from $m$ to $D$ if the data is forwarded along path $j$ after departing from $m$. Then the whole equation can be written as follows.

$$
\begin{aligned}
\mathrm{EDD} & =\sum_{m=1}^{K}\left(P_{S, m}^{c} \times\left(C_{S, m}+E\left(D_{m, D}\right)\right)\right) \\
& =\sum_{m=1}^{K}\left(\left(\prod_{h=0}^{m-1} P_{h, h+1}^{c}\right) \times\left(C_{S, m}+\sum_{j=1}^{N_{m}} P_{m}^{j} D_{m, D}^{j}\right)\right) .
\end{aligned}
$$

To calculate TTL, we assume $g(v)$, which is the vehicle's trajectory and obeys Gamma distribution [2]. Then given the expected vehicle delay $\mu_{i}$ and the variance of the vehicle delay $\delta_{i}$ between every two intersections along the transmission path, the total expected vehicle delay $E[V]$ and the variance of the vehicle delay $\operatorname{Var}[V]$ can be represented as $E[V]=$ $\sum_{i=1}^{N} \mu_{i}, \operatorname{Var}[V]=\Sigma_{i=1}^{N} \delta_{i}^{2}$, respectively.

$P[$ TTL $>$ EDD] is the probability of the target vehicle arriving at the receiving area later than transmitted data. Assuming that the data forwarding delay distribution and vehicle delay distribution are independent of each other, the delivery probability $P[\mathrm{TTL}>\mathrm{EDD}]$ can be computed as

$$
P[\mathrm{TTL}>\mathrm{EDD}]=\int_{0}^{\mathrm{TTL}} \int_{0}^{v} f(\rho) g(v) d \rho d v .
$$

$f(\rho)$ is the probability density function (PDF) of packet delay p. $g(v)$ is the PDF of vehicle delay $v$. Vehicles which are going to send data will firstly set a threshold for success ratio $\alpha$. Only those places satisfying $P$ [TTL $>$ EDD] $\geq \alpha$ with a minimum delivery delay can be chosen as the potential receiving area.

2.3. Network Topology and Path Arrangement. In this section, we will introduce the way to establish the network topology and the way to build the preliminary path from the source node to the destination node.

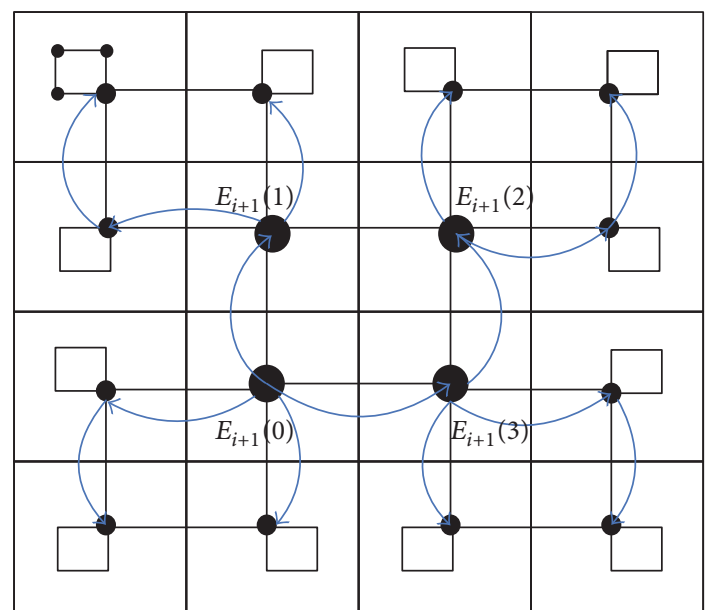

FIGURE 3: Hierarchical topology.

In order to arrange the network's topology better, it is reasonable to separate the whole map into many small regions with at least one $\mathrm{SN}$ in each of them responsible for receiving, temporarily holding, and forwarding data in the region and organize them in a hierarchical way. As shown in Figure 3, $E_{i(k)}(k=0,1,2,3)$ denotes the $k$ th $\mathrm{SN}$ in level $i$. And the arrows denote the possible delivery channels between two neighboring SNs. We assign four regions in level $i$ to form a bigger region in level $i+1$. One of the four SNs in level $i$ becomes the representative of them in level $i+1\left(E_{i+1(k)} \epsilon\right.$ $\left.E_{i(k)}\right)$, responsible for concentrating and disseminating data in level $i$. Then the topology of the network can be extended to higher levels in this way as shown in Figure 3.

As a simple example, Figure 4 shows a two-level hierarchical topology in $4 \times 4$ mesh. The red square is a basic region with a SN. The green square contains four basic regions and denotes level 1 . As a consequence, the blue square denotes level 2.

Also, in Figure 4, the dots which represent the Stationary Nodes are also called the Eyes; Eye theory [8] is aiming to find the best Total Communication Distance (TCD). TCD is the distance of data transmitted from the source node to the destination node, so min (TCD) can be the best solution. In 


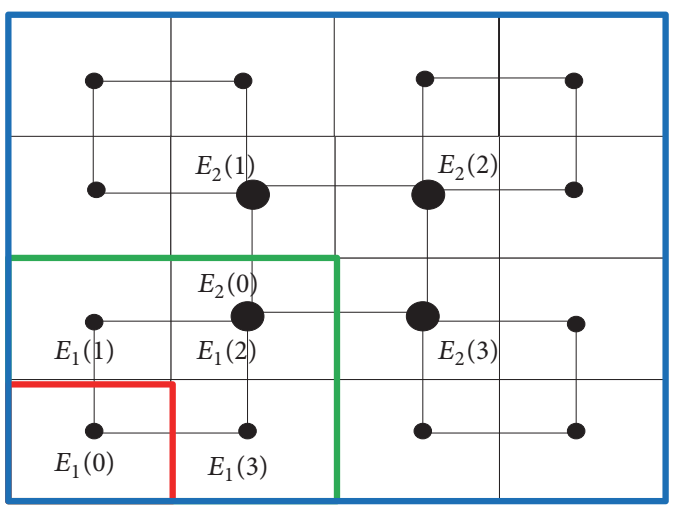

FIgURE 4: A simple example of hierarchical topology.

order to build a preliminary forwarding path with $\min$ (TCD) from the source node to the destination node, as shown in Figure 5, it is better to address SNs.

In a $k$ level VANET, we reserve $2 \mathrm{k}$ binary bits for node addressing. In every level, codes $00,01,10$, and 11 represent nodes at the 0th, 1st, 2nd, and 3rd subregion, respectively. For instance, in a four-level VANET, (11110000) denotes the SN located at the 0th subregion in the 1st level and the 2nd level and the 3rd subregion in the 3rd level and 4th level. Along the delivery path from the source node to the destination node, the next Stationary Node is determined by comparing current address with destination address. If their higher level addressing number is the same, which indicates they are in the same region from higher level perspective, there is no need to transmit to higher levels. For example, in Figure 5, there are four levels in the network. The address of the source node $S$ is (00000001). The destination node D locates at (10111110). Comparing S with D, the highest different level of their addresses is level four. So the data must be transmitted to level 2 first, then level 3, and finally A (10001110), which is in the same region as $\mathrm{D}$ on the 4 th level. Then the data will be transmitted downwards along the path from $\mathrm{A}$ to $\mathrm{D}$ via $\mathrm{B}$ and C. In another case, if the source node is R (10001100), the data can be transmitted directly to A, since their codes are only different in the first level.

Note that this path is only a preliminary path, served as a reference for data forwarding; the actual forwarding path may be adjustable according to each node's delay estimation.

\subsection{Diffusion Strategy. When in light-traffic conditions, users} may want to forward data to remote areas as quickly as possible. However, MNs which can be the next relay nodes are difficult to obtain in such cases. Inspired by DDB [9], our adaptive diffusion strategy used in this case will help quick forwarding. In diffusion mode, every potential relay node is introduced to Additional Area (the grey area in Figure 6) according to its current GPS position and the carrier's GPS position. And the Additional Area can be calculated by

$$
\mathrm{AC}(d)=2\left(\int_{-d / 2}^{1} \sqrt{1-x^{2}} d x\right.
$$

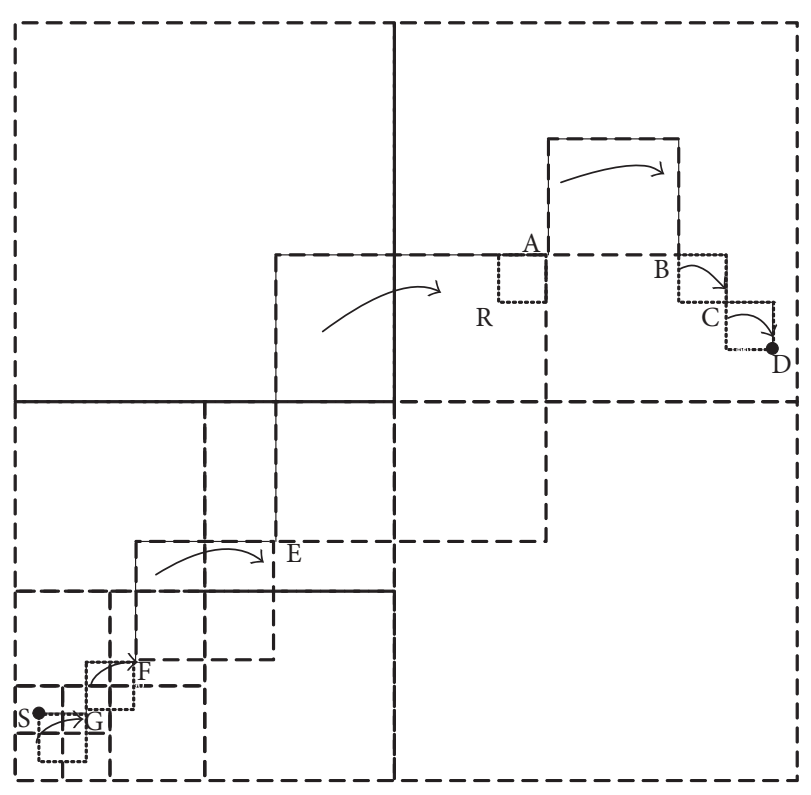

Figure 5: Forward path diagram.

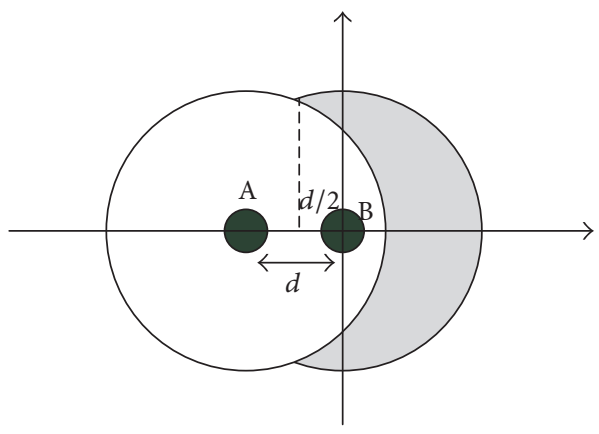

Additional Area

FIGURE 6: The increase range of schematic diagram.

$$
\begin{aligned}
& -\int_{-d / 2}^{-d+1} \sqrt{1-(x+d)^{2}} d x=\frac{d}{2} \sqrt{4-d^{2}} \\
& \left.+2 \arcsin \left(\frac{d}{2}\right)\right) .
\end{aligned}
$$

In order to forward data in a short time, the carrier always chooses the vehicle with the largest Additional Area as the next hop.

\section{THAF Protocol}

In this section, we explain the protocol of Trajectory-Based Hierarchical Adaptive Forwarding (THAF) as the following three steps. 
3.1. Construct the Network Topology. As shown in Figure 3, the network is divided into many small regions. And there should be at least one $\mathrm{SN}$ which is responsible for receiving, temporarily holding, and forwarding data in the region. Then the hierarchical regional network is built according to Section 2.3.

3.2. Data Forwarding. The preliminary transmission path will be initialized after calculating the receiving area based on delay estimation as we have discussed in Section 2.2. Based on the preliminary path, data are transmitted from the source vehicle to the destination one. Note that the preliminary path is just a reference; the real transmission path is not restricted to it. Furthermore, when there are conditions satisfying the case in our former descriptions in Section 2.4, diffusion strategy will be chosen automatically to reduce delay.

3.3. The Destination Node Receives the Data. The data are directly received if the destination vehicle just arrives in the receiving area. And otherwise, the data are sent to the destination vehicle along its trajectory in the opposite direction until encountering the destination vehicle. Note that the delivery process is a unicast data forwarding scheme; there is no need to keep a backup.

\section{Simulation}

4.1. Simulation Settings. In this section, we evaluate the performance of THAF. The evaluation setting is based on document [2], we have developed the simulation platform by $\mathrm{C}$ and $\mathrm{C}++$ language.

We build a $6.75 \mathrm{~km}$ (4.19 miles) $\times 6 \mathrm{~km}$ ( 3.73 miles $)$ simulation area with 36 intersections and construct a threelevel network which divides the subregion into $4 \times 4$. The length of each subregion is near to $1500 \mathrm{~m}$ (0.93 miles). Each region has a Stationary Node, so there are altogether 16 SNs. AP is located in central region, which connects with TCC directly. The parameters of the platform are shown in Table 1.

4.2. Experiment of Simulations. In the current state-of-theart schemes for $\mathrm{v} 2 \mathrm{v}$ transmission, only STDFS [3] is based on determined delivery delay estimation. And flooding is a scheme which will send data stream from one interface to the other interfaces. The simulation compares the performance of delivery delay and success ratio of THAF with that of STDFS and flooding under different traffic flow, vehicle speed deviation, and path deviation. When evaluating the performance of STDFS, we assume that every node has the capability to get nodes distribution knowledge in the network. And when evaluating the performance of flooding, we assume there is no transmission conflict and vehicles have infinite buffer to store packets.

(1) The Performance of the Algorithms under Different Traffic Flow. Different traffic situations affect the delay and success ratio of data delivery. As shown in Figure 7, our algorithm has less delivery delay and higher success ratio than STDFS. Especially in the case of extremely light-traffic situations,
TABLE 1

\begin{tabular}{|c|c|}
\hline Communication radius & $200 \mathrm{~m}$ (0.12 miles) \\
\hline Vehicle flow rate & $\begin{array}{l}\text { The number of the vehicles } \\
\text { within the specified range. The } \\
\text { default value is } 100\end{array}$ \\
\hline Vehicle velocity & $\begin{array}{l}\text { Vehicle velocity, } \\
v \sim N\left(\mu_{v}, \delta_{v}\right), \mu_{v} \text { is the average } \\
\text { velocity, the maximum velocity is } \\
\mu_{v}+3 \delta_{v} \text {, the minimum velocity } \\
\text { is } \mu_{v}-3 \delta_{v} \text {, and the default } \\
\text { velocity is }(40,7) \mathrm{MPH} \text {. }\end{array}$ \\
\hline $\begin{array}{l}\text { The reliability rate of the } \\
\text { user's request }(\alpha)\end{array}$ & $\begin{array}{l}\text { The reliability rate of the user's } \\
\text { request }(\alpha) \text {, the default rate is } 0.9\end{array}$ \\
\hline TTL (Time-to-Live) & $\begin{array}{l}\text { The vehicle's travel time for the } \\
\text { trajectory, the default TTL value } \\
\text { is } 1000 \mathrm{~s}\end{array}$ \\
\hline $\begin{array}{l}\text { Vehicle transmission path } \\
\text { length }\end{array}$ & $\begin{array}{l}d_{i j} \pm \delta_{i j}, d_{i j} \text { is the distance from } \\
\text { node } i \text { to node } j, \delta_{i j} \text { is the } \\
\text { additional road distance, } \\
l \sim N\left(\mu_{l}, \delta_{l}\right), \mu_{l} \text { is } d_{i j}, \sigma_{l} \text { is } \delta_{i j} \\
\text { and the default value is } 3 \mathrm{~km} \\
(1.86 \text { miles) }\end{array}$ \\
\hline
\end{tabular}

our algorithm can be more reliable. Experiment results show that, even in the condition of extremely low traffic flow, we can ensure $95 \%$ of the success ratio. This is mainly because the hierarchical topology and path arrangement method described in Section 2.3 are applied. The experiments show that our method has better adaptability and stability under low traffic flow.

(2) The Performance of the Algorithms under Different Vehicle Speed. Vehicle speed determines the node distribution as well as the carry delay during the delivery process. As shown in Figure 8, THAF can achieve lower delivery delay than STDFS, especially when vehicles are at low vehicle speed. This is because the hierarchical topology enables vehicles to forward data by the preliminary path, which is more stable and reliable compared with STDFS which forwards data by calculating the transmission path after each step. Furthermore, we want to point out that THAF can maintain the stability of the success ratio at different vehicle speed.

(3) The Performance of the Algorithms under Different Speed Deviation. Shown in Figure 9, the increase of deviation of vehicle speed leads to higher delivery delay and lower delivery ratio, but our method still gets higher performance than STDFS, especially when the speed deviation is greater than $8 \mathrm{MPH}$. The simulation results show that if detailed traveling information of individual vehicles can be employed as well as statistical traffic, packet forwarding can be more accurate and effective.

(4) The Performance of the Algorithms under Different Path Deviation. We randomly select the changing trajectories of the vehicles. The simulation results are shown in Figure 10. When the trajectory changes more than 7 times, THAF and STDFS have greater delay and less delivery ratio while the 

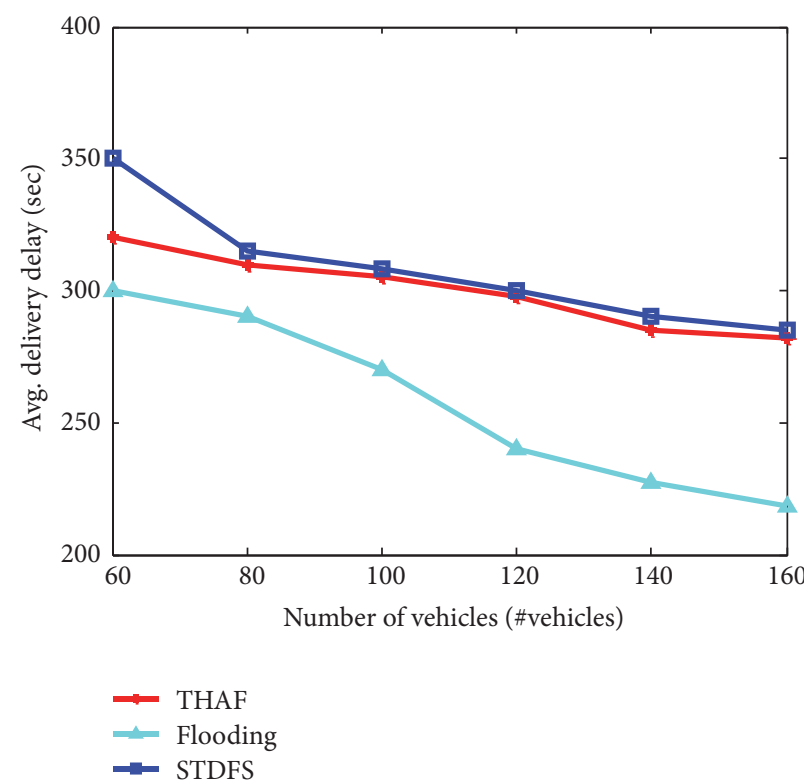

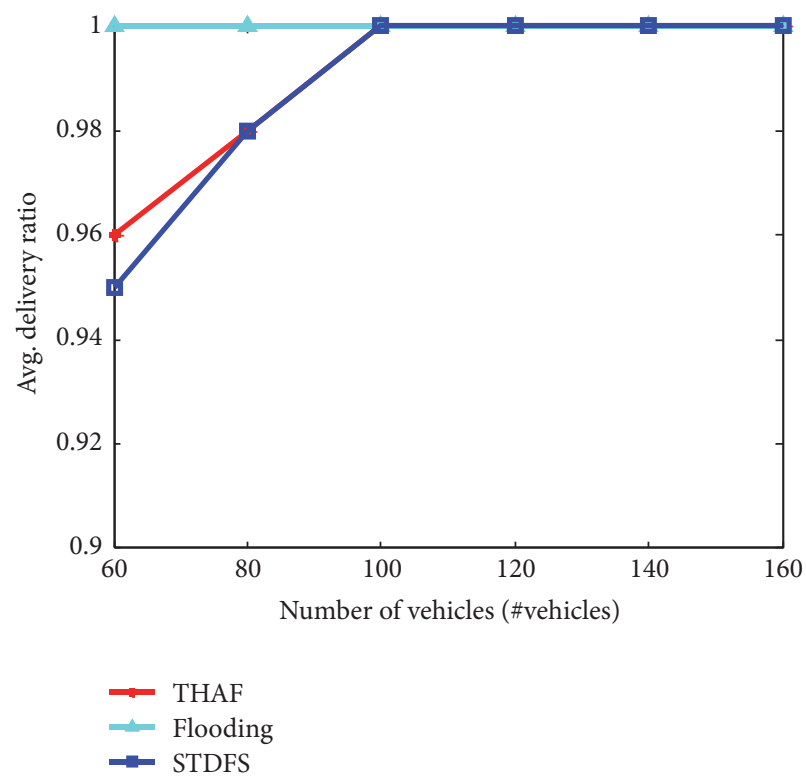

FIgURE 7: The performance of the algorithms under different traffic flow.
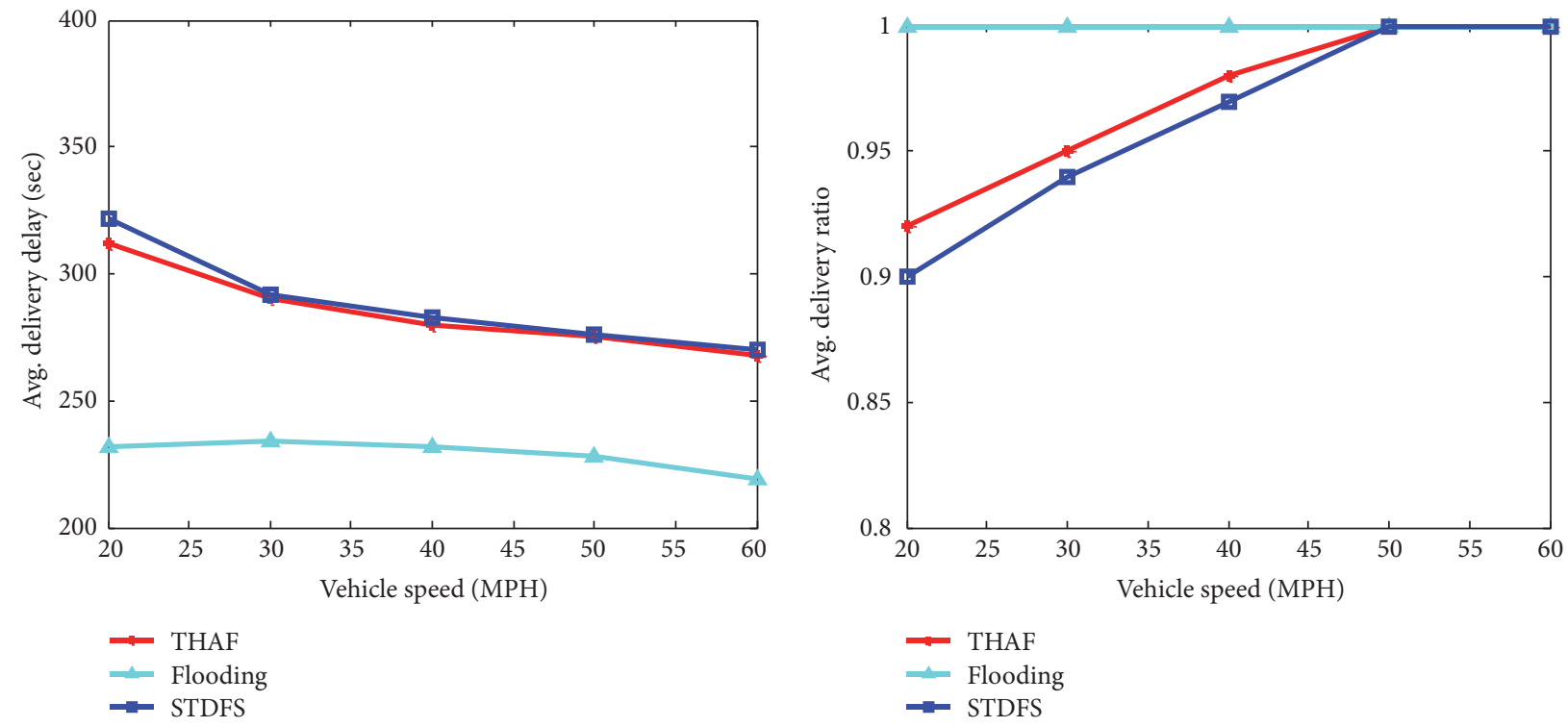

FIGURE 8: The performance of the algorithms under different vehicle speed.

flooding algorithm keeps stable all the time. The feasibility of our algorithm is superior to STDFS. STDFS is aiming to make a prediction of the whole network topology. So when a node changes its path, it cannot update in time. And this induces the low success ratio and more delay. THAF is mainly based on the principle of the subregion and adapts the actual vehicle trajectory in each subregion. So when the vehicle path changes, it can maintain the success ratio above 95 percent, as well as reduce the delivery delay.

In our experiments, flooding algorithm is dominant under various traffic flow and vehicle speed. As expected, flooding achieves the maximum delivery ratio and minimum delay in the network with the assumptions of infinite buffer and collision-free transmission. However, these assumptions are too ideal to be achieved in real situation. And according to IEEE DSCR standard, THAF is reasonable in practice.

\section{Conclusion}

In this paper, the Trajectory-Based Hierarchical Adaptive Forwarding scheme called THAF has been proposed by us in vehicular networks. And our goal is to provide a reliable and efficient vehicle-to-vehicle data delivery delay scheme by 

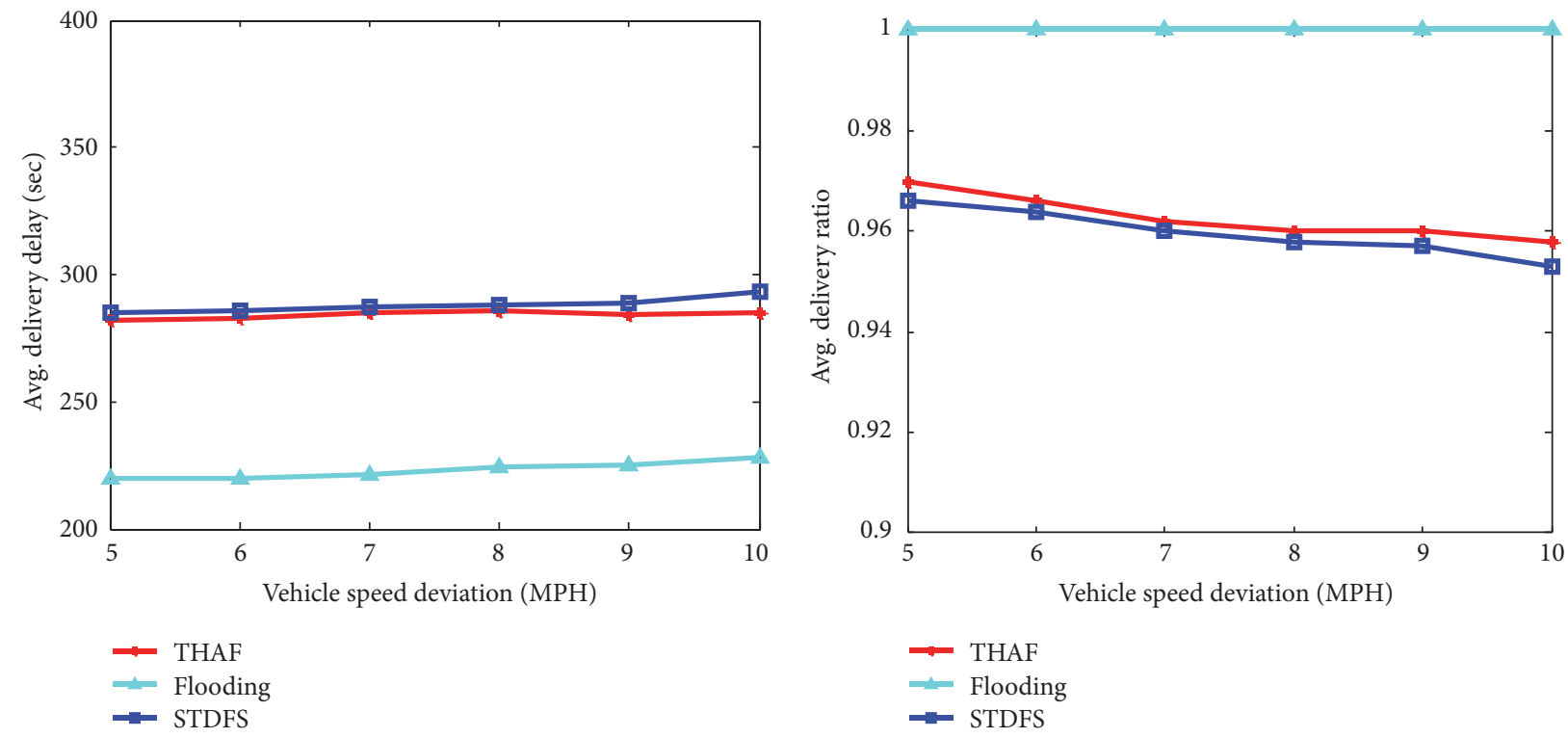

FIGURE 9: The performance of the algorithms under different speed deviation.
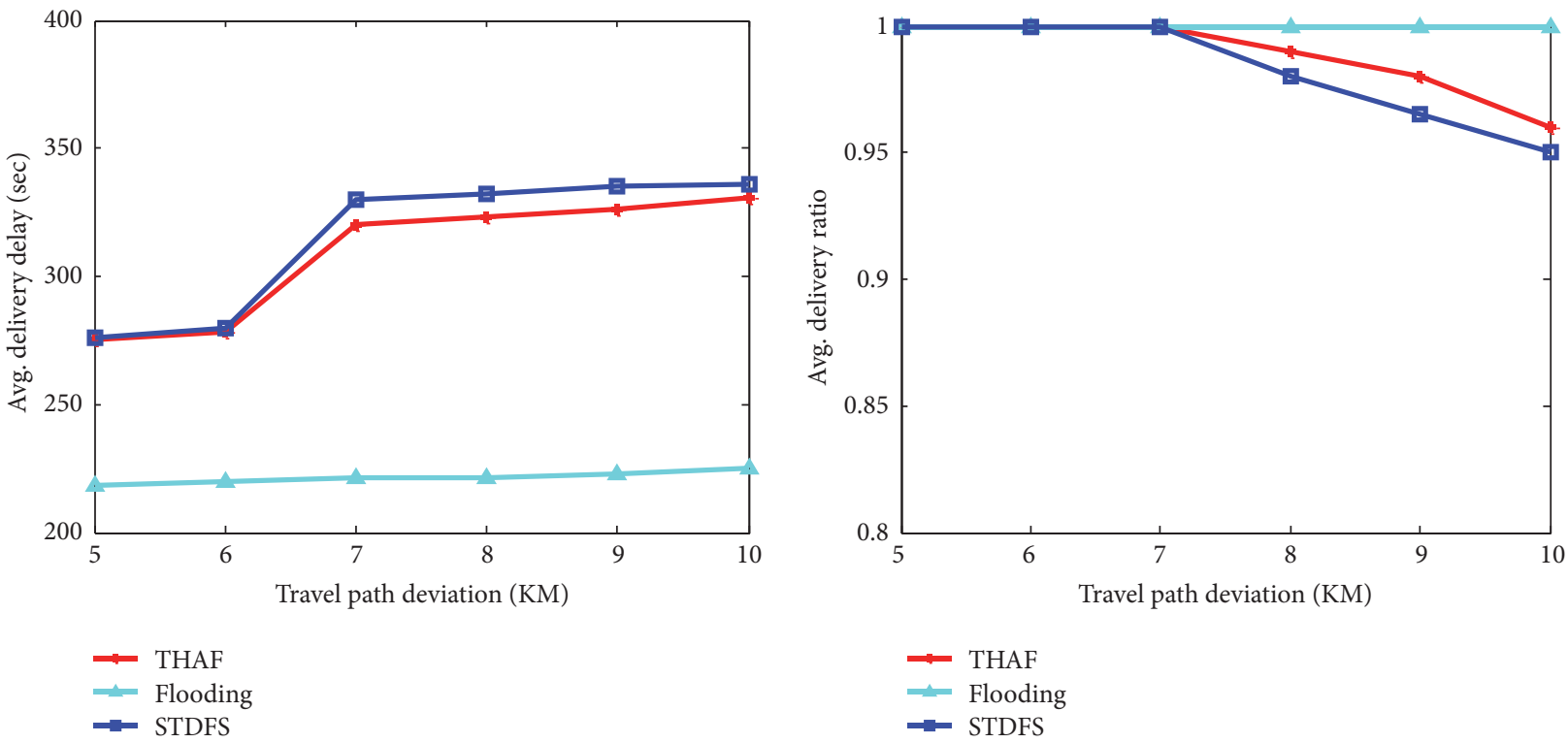

FIGURE 10: The performance of the algorithms under different path deviation.

minimizing the packet delivery delay subject to the required delivery probability. We achieve this goal by constructing a hierarchical network topology to optimize transmission path and apply adaptive diffusion strategy to forward data in lighttraffic situations. Experimental results show that THAF are able to effectively reduce the packet delivery delay and be suited to be applied in practice. As future work, we will investigate how to deploy the fixed nodes to further reduce the delivery delay.

\section{Competing Interests}

The authors declare that they have no competing interests.

\section{Acknowledgments}

This work is supported by National Science Foundation of China, under Grant 61373091; the Project of Science and Technology Department of Sichuan Province, under Grant 2016GZ0068; the National Science Foundation of China and the Civil Aviation Administration of China subsidization project, under Grant U1533203.

\section{References}

[1] A. A. Carter, "The status of vehicle-to-vehicle communication as a means of improving crash prevention performance," Tech. 
Rep. 01-19, National Highway Traffic Safety Administration, Washington, DC, USA, 2005.

[2] J. Jeong, S. Guo, Y. Gu, T. He, and D. H. C. Du, “Trajectorybased statistical forwarding for multihop infrastructure-tovehicle data delivery," IEEE Transactions on Mobile Computing, vol. 11, no. 10, pp. 1523-1537, 2012.

[3] F. Xu, S. Guo, J. Jeong et al., "Utilizing shared vehicle trajectories for data forwarding in vehicular networks," in Proceedings of the IEEE Conference on Computer Communications (INFOCOM '11), pp. 441-445, IEEE, April 2011.

[4] J. P. Jeong, T. He, and D. H. C. Du, "Trajectory based data forwarding schemes for vehicular networks," ZTE Communications, vol. 1, article 5, 2014.

[5] L. Y. Chen, S. T. Fu, J. Y. Zhang et al., “Trajectory-based optimal area forwarding for infrastructure-to-vehicle data delivery with partial deployment of stationary nodes," International Journal of Distributed Sensor Networks, vol. 2013, Article ID 929031, 10 pages, 2013.

[6] Y. Ding, C. Wang, and L. Xiao, "A static-node assisted adaptive routing protocol in vehicular networks," in Proceedings of the Fourth ACM International Workshop on Vehicular Ad Hoc Networks (VANET '07), pp. 59-68, Montreal, Canada, September 2007.

[7] Garmin, Garmin Traffic, http://www8.garmin.com/traffic/.

[8] S. Cang and J. Wu, "Minimizing total communication distance of a broadcast on mesh and torus networks," in Proceedings of the 1st Merged International Symposium on Parallel and Distributed Processing (IPPS/SPDP '98), Orlando, Fla, USA, April 1998.

[9] M. Heissenbüttel, T. Braun, M. Wälchli, and T. Bernoulli, "Optimized stateless broadcasting in wireless multi-hop networks," in Proceedings of the 25th IEEE International Conference on Computer Communications (INFOCOM '06), pp. 1-12, Barcelona, Spain, April 2006. 


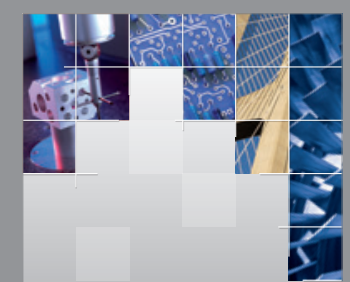

\section{Enfincering}
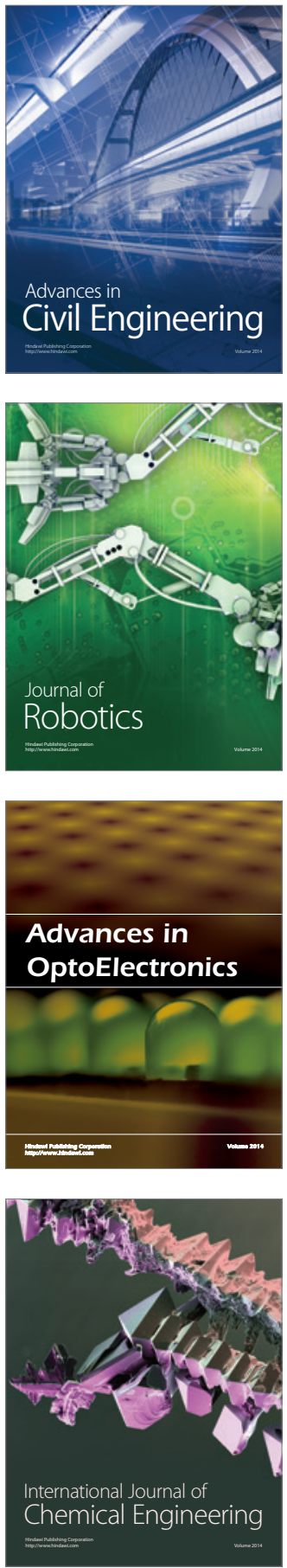

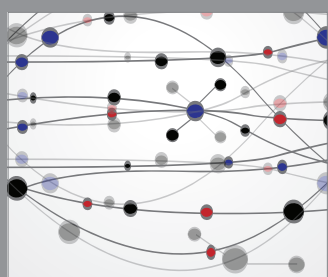

The Scientific World Journal

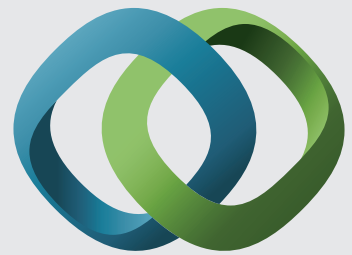

\section{Hindawi}

Submit your manuscripts at

https://www.hindawi.com
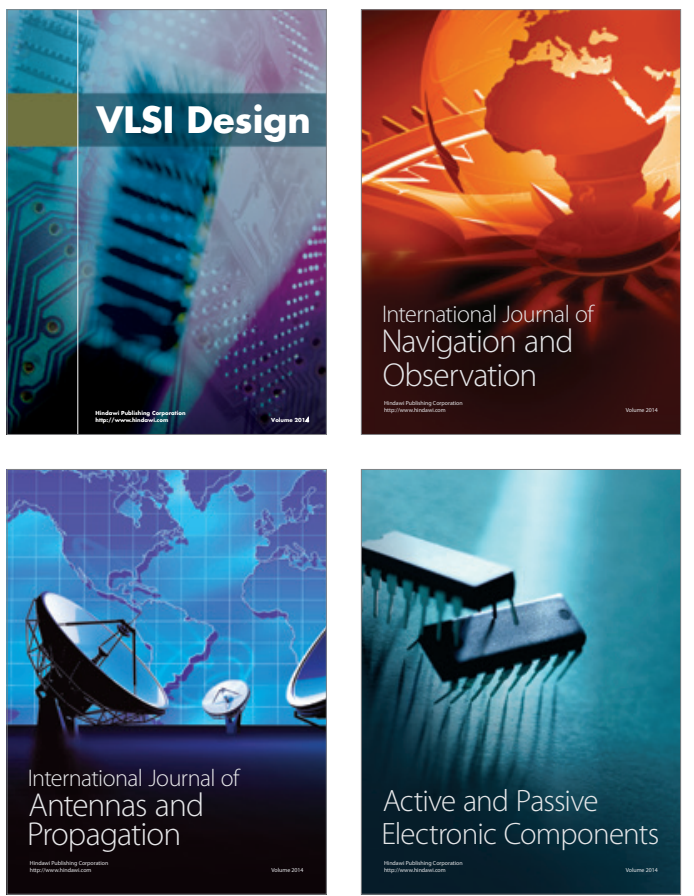
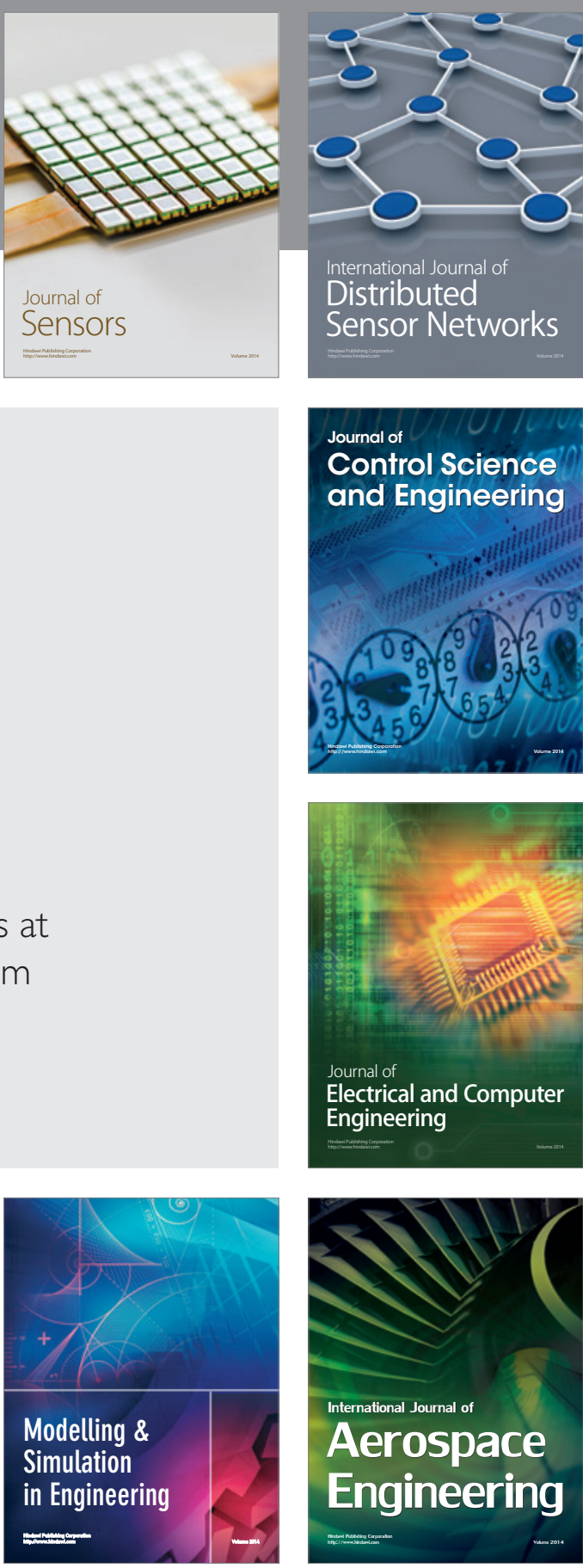

International Journal of

Distributed

Sensor Networks

$-$

Joumal of

Control Science

and Engineering
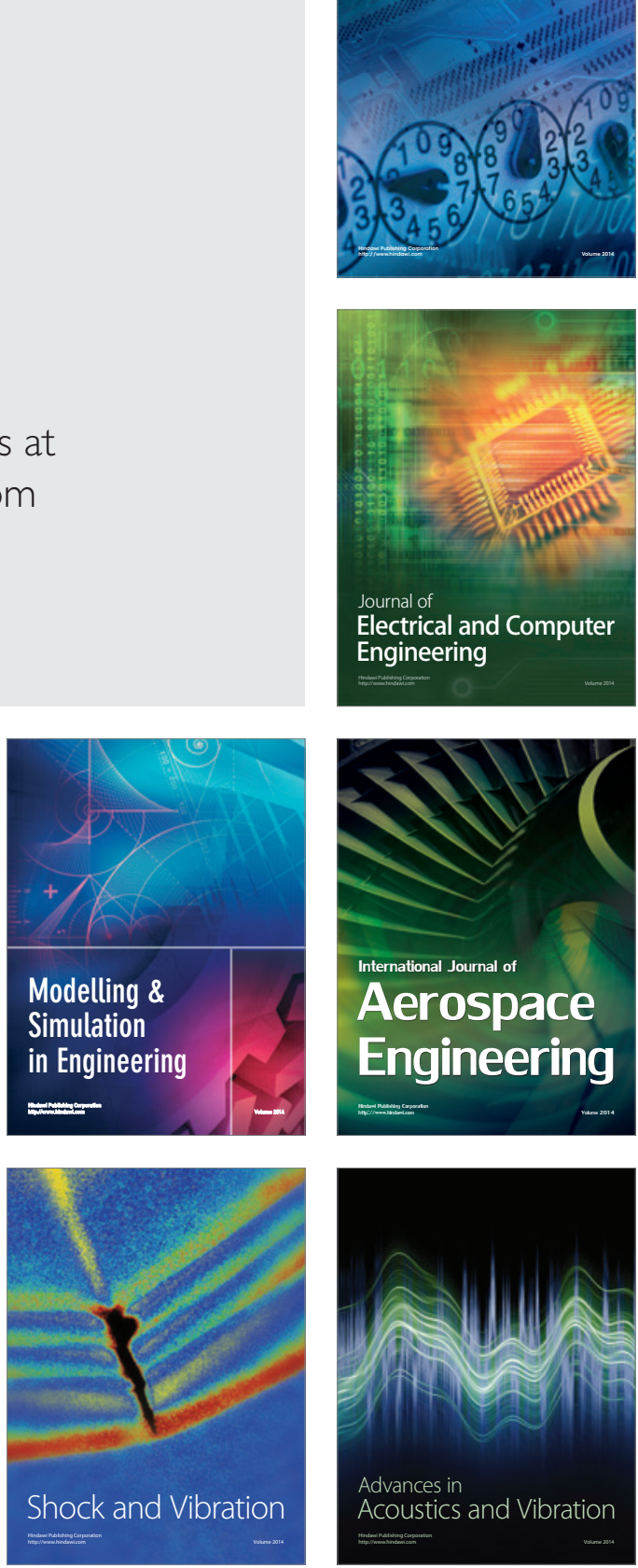